BULL. AUSTRAL. MATH. SOC.

VOL. 18 (1978), 439-446.

\title{
Composition operators
}

\section{R.K. Singh and B.S. Komal}

A study of centered composition operators on $l^{2}$ is made in this paper. Also the spectrum of surjective composition operators is computed. A necessary and sufficient condition is obtained for the closed unit disc to be the spectrum of a surjective composition operator.

\section{Preliminaries}

Let $L^{2}(\lambda)$ be the Hilbert space of all square integrable functions on a $\sigma$-finite measure space $(X, S, \lambda)$ and let $\phi$ be a non-singular measurable transformation from $X$ into itself. Then the equation $c_{\phi} f=f \circ \phi$ for every $f \in L^{2}(\lambda)$ defines a linear transformation. If $C_{\phi}$ happens to be a bounded operator on $L^{2}(\lambda)$, then we call it a composition operator. If $X=N$, the set of all non-zero positive integers and $\lambda$ is the counting measure on the family of all subsets of $N$, then $L^{2}(\lambda)=Z^{2}$, the Hilbert space of all square summable sequences.

In this note we have studied composition operators on $l^{2}$. The second section characterises centered composition operators while the third section is devoted to the study of the spectrum of a surjective composition operator. If $H$ is a Hilbert space, then $B(H)$ denotes the Banach algebra of all bounded linear operators on $H$.

\section{Centered composition operators on $z^{2}$}

Let $H$ be a complex Hilbert space, $T \in B(H)$, and let

Received 15 March 1978. 
$s_{T}=\left\{\left(T^{*}\right)^{k_{T} k}: k \in N\right\} \cup\left\{T^{k}\left(T^{*}\right)^{k}: k \in N\right\}$. Then $T$ is defined to be a centered operator if any two elements of $\boldsymbol{s}_{T}$ commute. These operators have been studied by Morrel and Muhly [4] in detail. We give a necessary and sufficient condition for a composition operator to be centered.

THEOREM 2.1. Let $\phi$ be a mapping from $N$ into itself such that $c_{\phi} \in B\left(z^{2}\right)$. Then $c_{\phi}$ is centered if and only if $f_{0}^{k}$ is constant on $\left(\phi^{p}\right)^{-1}(\{n\})$ for every $n \in N$ and $p \in N$, where $f_{0}^{k}$ is the Radon-Nikodym derivative of the measure $\lambda\left(\phi^{k}\right)^{-1}$ with respect to the measure $\lambda$.

For the proof of the theorem we need the following lemma.

LEMMA 2.2. If $\phi$ is a measurable transformation from a measure space $(X, S, \lambda)$ into itself such that $C_{\phi} \in B\left(L^{2}(\lambda)\right)$, then

$$
\left(C_{\phi}^{*}\right)^{k} C_{\phi}^{k}=M_{f_{0}^{k}} \text { for every } k \in N
$$

where $M_{f_{0}^{k}}$ is the multiplication operator induced by $f_{0}^{k}$.

Proof. Since $C_{\phi} \in B\left(L^{2}(\lambda)\right)$, it is easy to show that $C_{\phi} k=C_{\phi}^{k} \in B\left(L^{2}(\lambda)\right)$, where $\phi^{k}$ is obtained by composing $\phi k$-times. If $f$ and $g$ are any two elements in $L^{2}(\lambda)$ and $k \in N$, then

$$
\begin{aligned}
\left\langle\left(c_{\phi}^{*}\right)^{k} c_{\phi}^{k} f, g\right\rangle & =\left\langle c_{\phi}^{k} f, c_{\phi}^{k}\right\rangle \\
& =\left(c_{\phi^{k}} f, c_{\phi^{k}}^{g}\right\rangle \\
& =\int_{X} f \circ \phi^{k} \cdot \overline{g \circ \phi^{k}} d \lambda \\
& =\int_{X}(f \circ \bar{g}) \circ \phi^{k} d \lambda \\
& =\int_{X} f \circ \bar{g} d \lambda\left(\phi^{k}\right)^{-1}
\end{aligned}
$$




$$
\begin{aligned}
& =\int_{X} f \cdot \bar{g} \cdot f_{0}^{k} d \lambda \\
& =\left\langle M_{f_{0}^{k}} f, g\right\rangle .
\end{aligned}
$$

This shows that $\left(C_{\phi}^{*}\right)^{k} C_{\phi}^{k}=M_{f_{0}^{k}}$. Hence the proof of the lemma is complete.

Proof of theorem. Suppose that the condition of the theorem holds. Let $A, B \in{ }^{8} C_{\phi}$. Then $A=C_{\phi}^{*} C_{\phi}^{k}$ or $A=C_{\phi}^{2} C_{\phi}^{*}$ and $B=C_{\phi}^{*} C_{\phi}^{p}$ or $B=C_{\phi}^{m} C_{\phi}^{\star m}$ for some $k, \tau, p$, and $m$ in $N$. If $A=C_{\phi}^{*^{k}} C_{\phi}^{k}$ and $B=C_{\phi}^{* p} C_{\phi}^{p}$, then from the above lemma it follows that $A B=M f_{0}^{k^{M}} f_{0}^{p}=B A$. If $A=C_{\phi}^{*^{k}} C_{\phi}^{k}$ and $B=C_{\phi}^{m} C_{\phi}^{* m}$ and if $e^{(n)}$ is the $n$th basis vector defined by $e^{(n)}(q)=\delta_{n q}$ (the Kronecker delta), then

$$
\begin{aligned}
& (A B) e^{(n)}=C_{\phi}^{*}{ }^{k} C_{\phi}^{k} C_{\phi}^{m} C_{\phi}^{* m} e^{(n)} \\
& =M_{f_{0}^{k}} C_{\phi}^{m} e^{\left(\phi^{m}(n)\right)} \quad \text { (by definition of } C_{\phi}^{*}[8] \text { ) } \\
& =f_{0}^{k}(n) X_{\left\{\left(\phi^{m}\right)^{-1}\left(\left\{\phi^{m}(n)\right\}\right)\right\}} \text {, }
\end{aligned}
$$

where $X_{E}$ denotes the characteristic function of the set $E$. A similar computation shows that $B A e^{(n)}=f_{0}^{k}(n) X\left\{\left\{\phi^{m}\right)^{-1}\left(\left\{\phi^{m}(n)\right\}\right)\right\}$. Thus $A B=B A$. Suppose now that $A=C_{\phi}^{2} C_{\phi}^{*^{2}}$ and $B=C_{\phi}^{m} C_{\phi}^{*^{m}}$ and without loss of generality assume $m \leq 2$. Then 


$$
\begin{aligned}
A B e^{(n)} & =C_{\phi}^{Z} C_{\phi}^{*} C_{\phi}^{m} C_{\phi}^{*} e^{(n)} \\
& =C_{\phi}^{Z} C_{\phi}^{*^{2}-m_{M}} f_{0}^{m^{X}}\left\{\phi^{m}(n)\right\} \\
& =f_{0}^{m}\left(\phi^{m}(n)\right) C_{\phi}^{Z} C_{\phi}^{*^{2}-m_{X}}\left\{\phi^{m}(n)\right\} \\
& \left.=f_{0}^{m}\left(\phi^{m}(n)\right) X\left\{\phi^{2}\right)^{-1}\left(\left\{\phi^{2}(n)\right\}\right)\right\}
\end{aligned}
$$

Also

$$
\begin{aligned}
& B A e^{(n)}=C_{\phi}^{m} C_{\phi}^{*} C_{\phi}^{Z} C_{\phi}^{*} e^{(n)} \\
& =C_{\phi}^{m}{ }_{f_{0}^{m}} C_{\phi}^{Z-m_{C} C_{\phi}^{2}} e^{(n)} \\
& =C_{\phi^{M}}^{m} f_{0}^{m^{X}}\left\{\left(\phi^{2-m}\right)^{-1}\left(\left\{\phi^{2}(n)\right\}\right)\right\} \\
& =f_{0}^{m}\left(\phi^{m}(n)\right) x_{\left\{\left(\phi^{2}\right)^{-1}\left(\left\{\phi^{Z}(n)\right\}\right)\right\}} \text {. }
\end{aligned}
$$

This shows that $A B=B A$.

On the other hand, suppose the condition of the theorem is not true. Then there exist $n_{1}, n_{2} \in\left(\phi^{p}\right)^{-1}(\{n\})$ such that $f_{0}^{k}\left(n_{1}\right) \neq f_{0}^{k}\left(n_{2}\right)$ for some $p, k, n \in N$. If $A=C_{\phi}^{* k} C_{\phi}^{k}$ and $B=C_{\phi}^{p} C_{\phi}^{* p}$, then $A B e^{\left(n_{1}\right)}=f_{0}^{k} \cdot X_{\left\{\left(\phi^{p}\right)^{-1}\left(\left\{\phi^{p}\left(n_{1}\right)\right\}\right)\right\}}$ and $B A e^{\left(n_{1}\right)}=f_{0}^{k}\left(n_{1}\right) X_{\left\{\left(\phi^{p}\right)^{-1}(\{n\})\right\}}$. Since $f_{0}^{k}\left(n_{1}\right) \neq f_{0}^{k}\left(n_{2}\right)$, we can conclude that $A B \neq B A$. Hence $c_{\phi}$ is not centered. This completes the proof of the theorem.

\section{Spectrum of a composition operator on $l^{2}$}

This section is devoted to the study of the spectrum of a composition operator on $Z^{2}$. The set of all complex numbers will be denoted by $C$ and the set $D$ defined by $D=\{\lambda: \lambda \in C$ and $|\lambda| \leq 1\}$ is called the closed unit disc. The symbol $\sigma(T)$ stands for the spectrum of $T$. The 
unit circle will be denoted by $c$.

THEOREM 3.1. If $\phi: N \rightarrow N$ is an injection which is not a surjection, then $\sigma\left(C_{\phi}\right)=D$.

Proof. Since $\phi$ is not a surjection, there is an $n_{1} \in N$ such that $\lambda\left\{\phi^{-1}\left(\left\{n_{1}\right\}\right)\right)=0$. Let $\phi^{m}\left(n_{1}\right)=n_{m+1}$ for $m \in N$ and let $M=\operatorname{span}\left\{e^{\left(n_{m}\right)}: m \in N\right\}$. Then $M$ is a closed subspace of $l^{2}$. By the projection theorem $Z^{2}=M \oplus M^{\perp}$. Since $M$ is a reducing subspace of $C_{\phi}, C_{\phi}=\left.\left.C_{\phi}\right|_{M} \oplus C_{\phi}\right|_{M^{\perp}}$, where $\left.C_{\phi}\right|_{E}$ denotes the restriction of $C_{\phi}$ to the subspace $E$. Define the transformation $S$ from $Z^{2}$ into $M$ by

$$
S e^{(m)}=e^{\left(n_{m}\right)} \text { for every } m \in N \text {. }
$$

Then $S$ is a bounded linear transformation. Also $S$ is invertible and $\left.C_{\phi}\right|_{M}=S U^{*} S^{-1}$, where $U^{*}$ is the adjoint of the unilateral shift $U$. Since similar operators have the same spectrum [3, Problem 60], we have $\sigma\left(\left.C_{\phi}\right|_{M}\right)=\sigma\left(U^{*}\right)$. Thus $\sigma\left(\left.C_{\phi}\right|_{M}\right)=D$ by the solution to Problem 67 of [3]. Since $\sigma\left(C_{\phi}\right)=\sigma\left(\left.C_{\phi}\right|_{M}\right) \cup\left(\left.C_{\phi}\right|_{M}\right), D \subset \sigma\left(C_{\phi}\right)$. From a corollary to Theorem 2.1 of [8], $\left\|C_{\phi}\right\|=1$ and hence $\sigma\left(C_{\phi}\right) \subset D$. Thus $\sigma\left(C_{\phi}\right)=D$.

The following two theorems compute the spectrum of invertible composition operators. We know that $C_{\phi}$ is invertible if and only if $\phi$ is invertible [8, Theorem 2.2].

THEOREM 3.2. Let $C_{\phi} \in B\left(z^{2}\right)$ be invertible, and assume for every $n \in N$ there exists an $m \in N$ such that $\phi^{m}(n)=n$. Let $m_{n}=\inf \left\{m: \phi^{m}(n)=n\right\}$ and $Q=\left\{m_{n}: n \in N\right\}$. Then $\phi\left(C_{\phi}\right)=\underset{q \in Q}{U}\left\{\lambda: \lambda^{q}=I\right\}$.

Proof. For $q \in Q$, let $E_{q}=\left\{n: n \in N\right.$ and $\left.\phi^{q}(n)=n\right\}$. Then $M_{q}=\operatorname{span}\left\{e^{(p)}: p \in E_{q}\right\}$ is a reducing subspace of $C_{\phi}$. Since $\phi$ is 
invertible, the family $\left\{M_{q} \mid q \in Q\right\}$ is a disjoint orthogonal family of subspaces which spans $l^{2}$. Thus $l^{2}$ can be written as $l^{2}=\sum_{q \in Q} \oplus M_{q}$. Since $\phi^{q}=I$ on $E_{q}$, it follows that $I=\left.\sum_{q \in Q} \oplus I\right|_{M_{q}}=\left.\sum_{q \in Q} \oplus c_{\phi}^{q}\right|_{M_{q}}$. From this we can conclude that

$$
\begin{aligned}
& \sigma(I)=\overline{\cup_{q \in Q} \sigma\left[\left.c_{\phi}^{q}\right|_{M}\right]},[3, \text { p. 80] }
\end{aligned}
$$

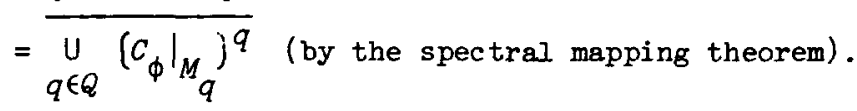

This shows that $\sigma\left(\left.c_{\phi}\right|_{M}\right)=1$ for every $q \in Q$. Hence

$\sigma\left(\left.C_{\phi}\right|_{M}\right)=\left\{\lambda: \lambda^{q}=1\right\}$. Since $C_{\phi}=\left.\sum_{q \in Q} \oplus C_{\phi}\right|_{M_{q}}$,

$\sigma\left(C_{\phi}\right)=\underset{q \in Q}{U} \sigma\left(\left.C_{\phi}\right|_{M_{q}}\right)$. Hence $\sigma\left(C_{\phi}\right)=\underset{q \in Q}{U}\left\{\lambda: \lambda^{q}=1\right\}$.

COROLLARY. If $\phi$ is periodic with period $m$, then $\sigma\left(C_{\phi}\right)=\left\{\lambda: \lambda^{m}=1\right\}$.

THEOREM 3.3. If $C_{\phi} \in B\left(Z^{2}\right)$ is invertible and if for some $n \in N$, there does not exist any $m \in N$ such that $\phi^{m}(n)=n$, then $\sigma\left(C_{\phi}\right)=c$.

Proof. Let $n_{0} \in N$ be such that $\phi^{m}\left(n_{0}\right) \neq n_{0}$ for all $m \in N$. For $m \in N$, let $n_{m}=\phi^{m}\left(n_{0}\right)$ and $n_{-m}=\left(\phi^{m}\right)^{-1}\left(\left\{n_{0}\right\}\right)$. If $E=\left\{n_{m}: m \in Z\right\}$, where $z$ is the set of all integers, and $z_{E}^{2}=\operatorname{span}\left\{e^{(p)}: p \in E\right\}$, then

$$
\tau^{2}=z_{E}^{2} \oplus \tau_{N \backslash E}^{2}
$$

If the transformation $S$ from $l^{2}(z)$ into $Z_{E}^{2}$ is defined as $S e^{(m)}=e^{\left(n_{m}\right)}$ for every $m \in Z$, then $S$ is a bounded linear invertible transformation and $\left.C_{\phi}\right|_{l_{E}^{2}}=S W^{*} S^{-1}$, where $W$ is the bilateral shift. 
Thus by [3, Problem 60], $\sigma\left(\left.c_{\phi}\right|_{2_{E}^{2}}\right)=\sigma\left(w^{*}\right)$. From Problem 68 of [3], $\sigma\left(W^{*}\right)=c$ and from the relation (1), we have $\sigma\left(\left.C_{\phi}\right|_{Z_{E}}\right) \subset \sigma\left(C_{\phi}\right)$. Since $C_{\phi}$ is invertible implies that $C_{\phi}$ is unitary [8, Theorem 2.3], it follows that $\sigma\left(C_{\phi}\right)=c$.

COROLLARY. $\quad C_{\phi}$ is hermitian if and only if $\sigma\left(C_{\phi}\right) \subset\{-1,1\}$.

Proof. Suppose $C_{\phi}$ is hermitian. Then by Theorem 3 of [6], $\phi \circ \phi=I$, and hence $\sigma\left(C_{\phi}\right) \subset\{-1,1\}$ by the corollary to Theorem 3.2.

Conversely, if $\sigma\left(C_{\phi}\right) \subset\{-1,1\}$, then $C_{\phi}$ is invertible and so it is normal. Hence $C_{\phi}$ is hermitian in view of Corollary 1.7 of [5].

COROLLARY. Let $\phi: N \rightarrow N$ be an injection. Then $\sigma\left(C_{\phi}\right)=D$ if and only if $C_{\phi}$ is not an injection.

Proof. Suppose $c_{\phi}$ is not an injection. Then $\phi$ is not onto. Thus by Theorem 3.1, $\sigma\left(C_{\phi}\right)=D$.

On the other hand, if $C_{\phi}$ is an injection, then $\phi$ is onto. This shows that $\phi$ is invertible which further shows that $C_{\phi}$ is unitary. Hence $\sigma\left(C_{\phi}\right) \subset c$, which is a contradiction.

\section{Bibliography}

[1] Ronald G. Douglas, Banach algebra techniques in operator theory (Pure and Applied Mathematics, 49. Academic Press, New York and London, 1972).

[2] Paul R. Halmos, Measure theory (Van Nostrand, New York, Toronto, London, 1950. Reprinted: Graduate Texts in Mathematics, 18. Springer-Verlag, New York, Heidelberg, Berlin, 1974). 
[3] Paul R. Halmos, A Hilbert space problem book (Van Nostrand, Princeton, New Jersey; Toronto; London; 1967. Reprinted: Graduate Texts in Mathematics, 19. Springer-Verlag, New York, Heidelberg, Berlin, 1974).

[4] Bernard B. Morrel and Paul S. Muhly, "Centered operators", Studia Math. (to appear).

[5] Heydar Radjavi, Peter Rosenthal, Invariant subspaces (Ergebnisse der Mathematik und ihrer Grenzgebiete, 77. Springer-Verlag, Berlin, Heidelberg, New York, 1973).

[6] Raj Kishor Singh, "Normal and hermitian composition operators", Proc. Amer. Math. Soc. 47 (1.975), 348-350.

[7] R.K. Singh, "Composition operators induced by rational functions", Proc. Amer. Math. Soc. 59 (1976), 329-333.

[8] R.K. Singh and B.S. Komal, "Composition operator on $\imath^{p}$ and its adjoint", Proc. Amer. Math. Soc. (to appear).

Department of Mathematics,

University of Jammu,

Jammu,

India. 